\title{
Developing Kernel and Rachis Node Induce the Trichothecene Pathway of Fusarium graminearum During Wheat Head Infection
}

\author{
Peter Ilgen, Birgit Hadeler, Frank J. Maier, and Wilhelm Schäfer \\ Biocenter Klein-Flottbek, Molecular Phytopathology and Genetics, University of Hamburg, Ohnhorststrasse 18, \\ 22609 Hamburg, Germany
}

Submitted 17 November 2008. Accepted 16 March 2009.

The fungal pathogen Fusarium graminearum is the most common agent of Fusarium head blight (FHB) in small grain cereals and cob rot of maize. The threat posed by this fungus is due to a decrease in yield and, additionally, mycotoxin contamination of the harvested cereals. Among the mycotoxins, trichothecenes influence virulence of $\boldsymbol{F}$. $\mathrm{gra}$ minearum in a highly complex manner that is strongly host- as well as chemotype-specific. The factors inducing mycotoxin production during plant infection are still unknown. To evaluate the induction of the trichothecene pathway, the green fluorescence protein $(G F P)$ gene was fused to the promoter of the TRI5 gene coding for the trichodiene synthase and integrated into the genome by homologous integration. The resulting mutant contains a fully functional TRI5 gene ensuring virulence on wheat and exhibits GFP driven by the endogenous TRI5 promoter. We are now able to monitor the induction of trichothecenes under real-time conditions. To localize the fungus in the plant tissue, the $d s R e d$ gene was integrated under constitutive control of the glycerol-3-phosphate dehydrogenase $(\operatorname{gpd} A)$ promoter. We are now able to show that, first, induction of GFP as well as trichothecene production in the reporter strain reflects TRI5 induction and trichothecene production in the wild type; second, expression of TRI5 is inducible during growth in culture; and, third, trichothecene production is not uniformly induced during the onset of infection but is tissue specific during fungal infection of wheat.

Fusarium graminearum is the main causal agent of Fusarium head blight, a disease that leads to severe loss of yield and quality of the grain (Desjardins et al. 1996; Goswami and Kistler 2004). In addition to that, mycotoxins produced by the fungus can accumulate in foodstuffs and cause healththreatening toxication of humans and animals (Marasas et al. 1984). Therefore, several countries have set maximum levels for mycotoxin contamination in cereals and their products; for example, the European Union set the tolerable daily intake (TDI) value for the trichothecene deoxynivalenol (DON) to $1 \mu \mathrm{g} / \mathrm{kg}$ of body mass (Commission Regulation [EC] no. $1881 / 2006$ of 19 December 2006), and the United States Food and Drug Administration announced the advisory level of DON at $1 \mathrm{ppm}$ in finished wheat products (United States

Corresponding author: W. Schäfer; Telephone: ++ 49 (0)40 42816 266; Fax: ++ 49 (0)40 42816 513; E-mail: Schaefer@botanik.uni-hamburg.de
Department of Health and Human Services 1993). The mycotoxins produced by $F$. graminearum include the group of type B trichothecenes and, in this group, DON and nivalenol (NIV) as well as their acetylated derivatives (4ANIV, $3 \mathrm{ADON}$, and 15ADON) show a high abundance in Fusarium spp.-infected cereals (Desjardins et al. 1993). The toxicity of these compounds is believed to result from the ability to bind to the $60 \mathrm{~S}$ ribosomal subunit, thus leading to an inhibition of protein synthesis and an induction of apoptosis (Rocha et al. 2005). Over the past 20 years, the trichothecene biosynthesis pathway was under heavy investigation and, today, the complete TRI gene cluster is known (Kimura et al. 2007). It consists of seven pathway genes necessary for the synthesis of DON and its derivatives, two genes responsible for the regulation (TRI6 and TRI10) and one transporter gene (Desjardins et al. 1993; Kimura et al. 2007; Proctor et al. 1995b; Tag et al. 2001). The first dedicated enzyme of the biosynthetic pathway, the trichodiene synthase encoded by the TRI5 gene, is of special interest. Recent studies on mutants exhibiting a disrupted trichodiene synthase ( $\triangle T R I 5)$ show that these mutants have a dramatically reduced virulence on wheat and are no longer able to colonize the floret (Hohn and Desjardins 1992; Maier et al. 2006; Proctor et al. 1995a). The $\Delta T R I 5$ mutant can still colonize the inoculated wheat spikelet but is not able to protrude into the rachis, and its growth is confined to the point of inoculation. Whereas DON and NIV have been shown to be host-specific virulence factors for the infection of wheat, they seem to be of minor importance for a successful infection of maize and barley (Jansen et al. 2005; Maier et al. 2006). It is yet unclear which factors lead to the induction of the trichothecene synthesis during infection and why the pathway is induced, although the toxin is not needed for a successful colonization of maize cobs. A better understanding of the ecological and physiological relevance of mycotoxins is needed to provide new starting points for the development of resistant crops or specific inhibitors to decrease mycotoxin contamination by $F$. graminearum. Up to now, the methods of choice were restricted to reverse-transcriptase polymerase chain reaction (RT-PCR) to determine the transcript levels of trichothecene-related genes as well as time- and cost-extensive high-performance liquid chromatographymass spectrometry (HPLC-MS)-assisted measurements of DON contamination. To promote research in this issue, we developed a reporter strain of $F$. graminearum expressing the green fluorescence protein $(G F P)$ gene under control of the endogenous promoter of TRI5, thus giving us a potent bioimaging tool to monitor induction of the trichothecene pathway during plant infection. 


\section{RESULTS}

Generation of the $F$. graminearum TRI-pathway induction reporter strain.

We wanted to detect mycotoxin inducing-substances during in culture growth and monitor the inducibility of the mycotoxin production during wheat head infection. Therefore, the promoter of the initial gene of the trichothecene pathway was fused to the GFP gene. Additionally, the $d s R e d$ gene under the control of the constitutive glycerol-3-phosphate dehydrogenase promoter $(g p d A)$ was expressed to demonstrate fungal mycelium during plant infection. This allows differentiating between fungal growth and mycotoxin induction. To rule out any genome localization effects, the aim was to obtain an $F$. graminearum strain only affected in one genomic locus but with three features: i) a functional TRI5 locus, ii) a TRI5 promoter that regulates the transcription of $G F P$, and iii) a constitutively expressed $d s R e d$ gene. To obtain the first two features, a vector carrying the GFP-coding region under the control of the TRI5 promoter was constructed: A 1,095-bp fragment of the TRI5 gene locus was amplified with primers TRI5Prom-fwd binding 945 bp upstream and TRI5Prom-rev binding $150 \mathrm{bp}$ downstream of the TRI5 start codon. This fragment covers nearly the complete sequence upstream of the TRI5 start codon and is thought to include the complete TRI5 promoter. The GFP-coding region was derived from plasmid pIGPAPA, with primers TRI5-GFP-fwd and GFPHindIII-rev introducing a HindIII restriction site into the PCR product by using plasmid pIGPAPA as a template. The TRI5 promoter fragment and the GFP product were combined and amplified by fusion PCR (Amberg et al. 1995; Jansen et al. 2005) with primers TRI5Prom-fwd and GFPHindIII-rev and cloned into the pGEM-T cloning vector. Finally, the TRI5promoter::GFP fragment was isolated from the pGEM-T backbone by HindIII-ScaI double digestion and ligated to HindIII-EheI-cut pAN7-1 which carries the hygromycin phosphotransferase $(h p h)$ gene under the control of the gpdA promoter and confers resistance to the antibiotic hygromycin. The resulting vector was designated pTRI5:: GFP (Fig. 1A). The vector was linearized in the promoter sequence with Acc65I and integrated into the genome of $F$. graminearum isolate $\mathrm{Fg} 8 / 1$ by homologous recombination (Fig. 1A) via protoplast-mediated transformation (Maier et al. 2006). Finally, to obtain the third feature, the EcoRIHindIII fragment from pPgpd-dsRed, carrying the gpdA promoter-driven $d s R e d$ gene, was transferred into pII99, thus creating plasmid pII99DsRed. Plasmid pII99 carries the dominant selection marker $n p t I I$, coding for geneticin resistance under control of the $g p d A$ promoter, which is needed as a second selection marker. A verified transformant from the first transformation was transformed with plasmid pII99dsRed. To target this vector into the backbone of the already integrated pTRI5::GFP, the pII99dsRed plasmid was linearized with $P v u \mathrm{I}$ in the ampicillin resistance gene prior to transformation (Fig. 1B). The resulting reporter strain was named TRI5prom::GFP.

\section{Molecular characterization of the reporter strain.}

After transformation, positive mutants were picked from the selection plates and characterized via Southern blot analysis (Fig. 2). Genomic DNA was cut with restriction enzymes Eco81I and CpoI to gain a unique pattern of fragments during gel electrophoresis. Strain no. 864.6.1 from the first transformation (integration of pTRI5::GFP) was chosen to be transformed with pII99dsRed to create the final mutant. Two colonies resistant to geneticin and showing $d s R e d$ fluorescence on agar plates were selected for further analysis (904.8.1 and 905.7.1).

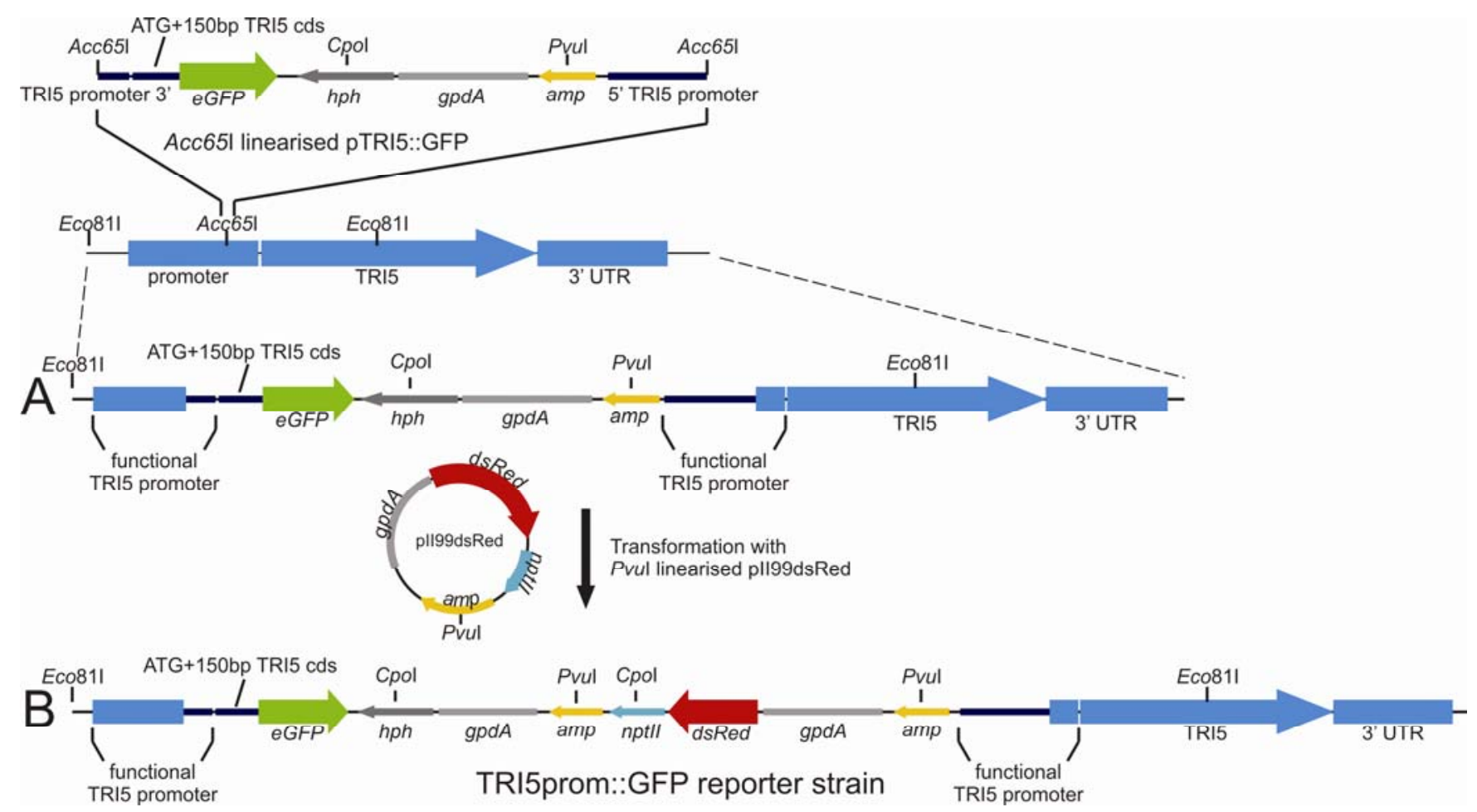

Fig. 1. Schematic illustration of the integration events that create the final mutant TRI5prom::GFP with $d s R e d$. A, Vector pTRI5::GFP carrying the TRI5 promoter-green fluorescent protein $(G F P)$ fusion construct was linearized with restriction enzyme Acc65I in the TRI5 promoter sequence, resulting in a single crossover with the targeted genomic sequence. This leads to a duplication of the TRI5 promoter by reassembling of the native sequence with the vector sequence. Both the TRI5 gene and the integrated GFP sequence are now under the control of the endogenous TRI5 promoter. B, Prior to transformation, the plasmid pII99dsRed was linearized in the amp sequence with $P v u \mathrm{I}$, resulting in a targeted integration into the backbone of pTRI5::GFP already present in the genome. These transformations led to the final reporter strain with inducible GFP and constitutive $d s R e d$ expression. 
$G F P$ reporter strain exhibits wild-type-like behavior during growth, virulence, gene expression, and DON production.

The TRI5prom::GFP strain showed the same growth patterns on different media (solid and liquid) as the wild type (data not shown). Virulence of the reporter strain was tested during infections of wheat heads of cv. Nandu. The strain developed the same symptoms as the wild type and, 3 weeks after infection, the infected spike was bleached completely (Fig. 3). The addition of ammonium sulfate to the medium was previously described to stimulate TRI5 expression and DON production (Covarelli et al. 2004; Lemmens et al. 2004). Therefore, we chose ammonium sulfate (AS) to investigate the induction of DON production and expression of trichothecene clusterrelated genes in the TRI5prom::GFP strain under in vitro conditions in 96-well plates. Mycelium was collected 4 days after inoculation (dai) and RNA was extracted from 96-well plate cultures of wild-type $F$. graminearum and the $G F P$ reporter strain grown in minimal medium (MM) without (control, noninducing conditions) and with (inducing conditions) the addition of $0.2 \mathrm{M}$ AS. To equal the amounts of cDNA in the subsequent PCR reactions, the abundance of the $\beta$-tubulin PCR product was chosen as internal control. Gene expression levels for selected genes of the trichothecene gene cluster were estimated by RT-PCR with RNA samples of the wild type and the mutant. The results presented in Figure 4B show an increased expression level of the pathway genes TRIS and TRI4 under the influence of $0.2 \mathrm{M}$ AS. TRI4 encodes a cytochrome P450 monooxygenase, which is the second enzyme in the trichothecene pathway after the initial trichodiene synthase (Tokai et al. 2007). The transcript levels of both genes show the same behavior in the wild type as well as in the GFP reporter strain. These results clearly confirm the wild-type like character of the TRI5prom::GFP reporter strain as well as the inducing capabilities of AS under the conditions tested. There is an obvious link between the expression of TRI5 and the expression of $G F P$, indicating the correlation between TRI5 induction and the actual observed GFP fluorescence under the microscope. Surprisingly, neither TRI6 nor TRI10 show a significant difference in their transcription levels under inducing or noninducing conditions in the used system. Both genes are described as regulating factors of the trichothecene gene cluster (Proctor et al. 1995b; Tag et al. 2001). To investigate whether the increased TRI5 gene expression level corresponds to an increased produc- tion of DON, the toxin content in the remaining medium was measured with the RIDASCREEN DON enzymatic immunoassay (Fig. 4C). DON measurements of the 96-well plate culture samples 4 dai show that there is no significant toxin production in $\mathrm{MM}$ in either the wild type $(2.1 \mathrm{ppb})$ or the reporter strain $(2.2 \mathrm{ppb})$. Addition of $0.2 \mathrm{M}$ AS to the medium leads to a significant increase in DON concentration and results in an approximately 18 times higher concentration of DON than in MM without AS. This dramatic increase in toxin concentration can be related to the increase in TRI5 gene transcripts under the influence of AS (Fig. 4B).

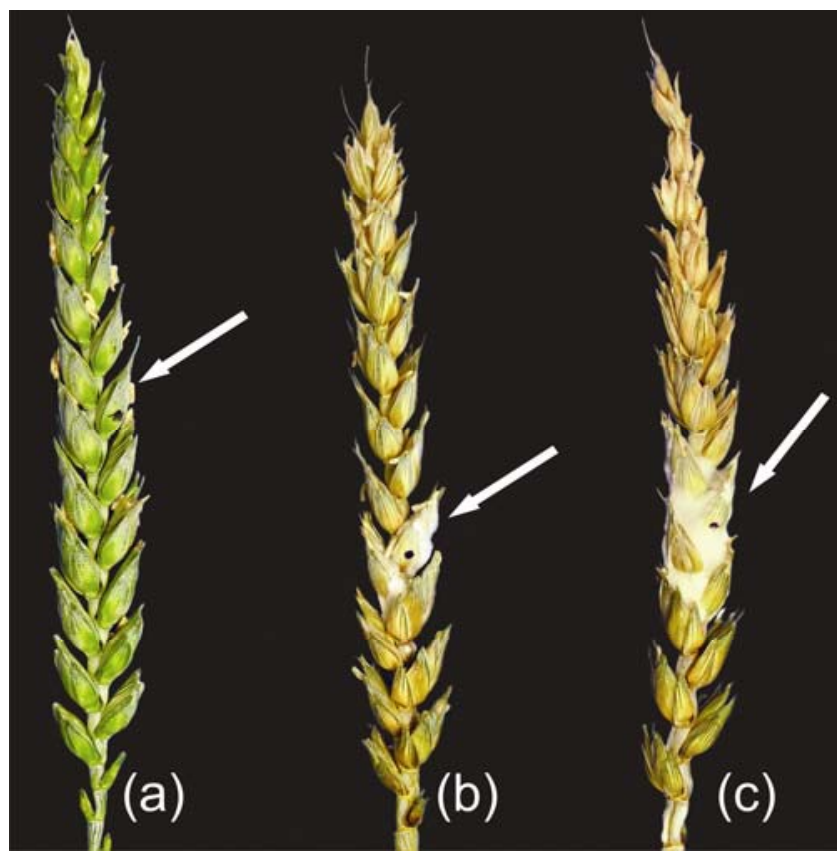

Fig. 3. Infection test on wheat plants of cv. Nandu, pictures showing infected wheat spikes at 21 days after inoculation. Florets were inoculated with 200 conidia in $10 \mu \mathrm{l}$ of $\mathrm{H}_{2} \mathrm{O}$ at anthesis; inoculated spikes were covered with a moistened plastic bag to ensure high humidity for the first 3 days of inoculation. Mock inoculation with water (control) (a), TRI5prom:: GFP mutant (b), and wild-type Fusarium graminearum (c). After 21 days of infection, the wild type as well as the TRI5prom::GFP strain show typical Fusarium head blight symptoms, proving wild-type-like character of the reporter strain during plant infection.

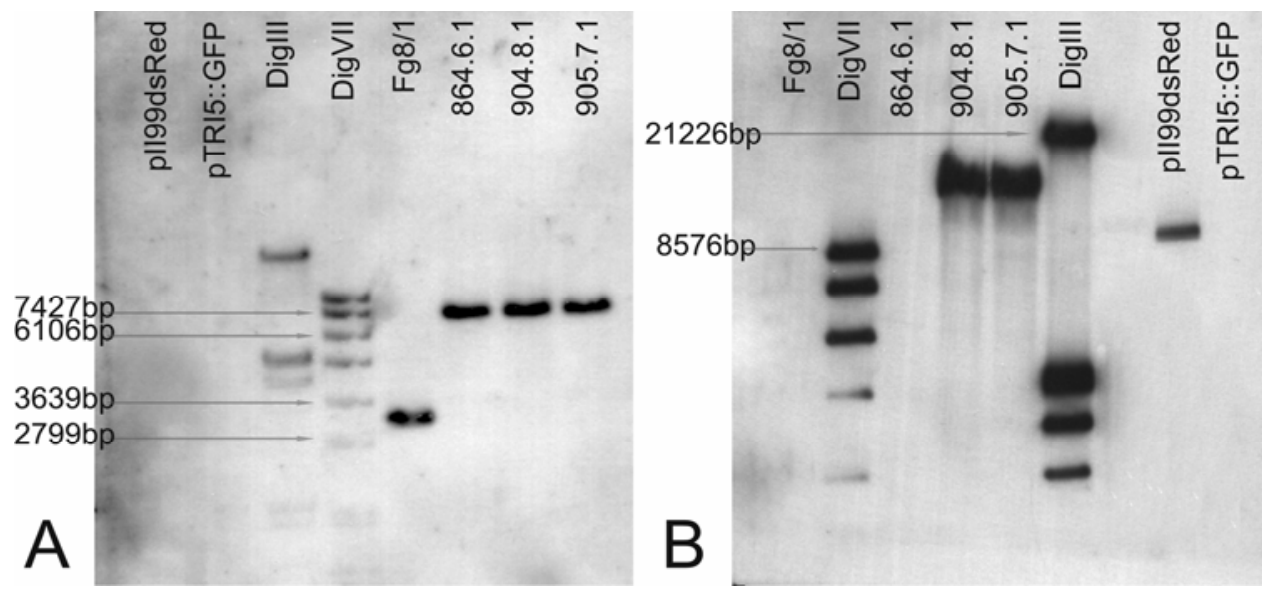

Fig. 2. Southern blot analysis of selected transformants after the integration of $d s R e d$-containing plasmid pII99dsRed. A, Hybridization with a TRI5 probe binding in the TRI5 coding sequence. No hybridization with the $d s R e d$ gene containing vector pII99dsRed or the TRI5 promoter-green fluorescent protein $(G F P)$ construct in pTRI5::GFP was observed. For the wild type, a band of 3,083 bp was expected. A shift to 7,008 bp shows integration of pTRI5::GFP into the TRI5 locus. B, Hybridization with a $d s R e d$ probe. Neither the wild type nor the primary mutant no. 864.6 .1 show hybridization with the probe but a band of $10 \mathrm{kbp}$ was expected for an integration of the 9,042-bp pII99dsRed into the backbone of the already present pTRI5::GFP and is visible in transformant no. 904.8.1 as well as in no. 905.7.1; transformant no. 905.7.1 was chosen for further experiments. 


\section{Bioimaging TRI5 expression} with an in vitro induction assay.

In addition to the already mentioned AS, several chemicals were tested for their ability to induce the trichothecene pathway. We chose three different phenolic compounds that are involved in the shikimate pathway and can be found in the plant cell wall in differing concentrations (Klepacka and Fornal 2006). In addition to these compounds, hydrogen peroxide was tested in our 96-well plate induction assay (Table 1). The addition of AS to the MM dramatically increased DON production and, in relation to that, the transcripts of trichothecene pathway genes were clearly elevated (Fig. 4). Within the tested range of $1 \mathrm{mM}$ to $0.5 \mathrm{M}$, the highest induction of GFP occurred between 0.1 and $0.2 \mathrm{M}$; therefore, the concentration of $0.2 \mathrm{M}$ AS was chosen for further experiments. At higher concentrations, GFP was still expressed but fungal growth was inhibited. First fluorescence signals of the GFP were visible $48 \mathrm{~h}$ after inoculation and, after 3 days of cultivation, every miniculture showed hyphae expressing GFP. Interestingly, the GFP induction was not distributed equally throughout the mycelium but was restricted to hyphae growing at the surface of the medium having contact with air as well as with the medium (Fig. 4A). In addition to that, the mycelium grew very densely, with short hyphae and a lot of branches. All tested phenolic compounds were severely inhibiting growth at a concentration higher than $1 \mathrm{mM}$ and germination of conidia was totally inhibited at the tested $5 \mathrm{mM}$ concentration. However, none of the phenolic acids was able to induce expression of TRI5 under the conditions tested. Formation of reactive oxygen species, like hydrogen superoxide, is a common hypersensitive response (HR) of plants to a pathogenic threat. Moreover, it was described that a concentration of $0.5 \mathrm{mM} \mathrm{H}_{2} \mathrm{O}_{2}$ positively influences the formation of DON in liquid cultures of $F$. graminearum (Ponts et al. 2006, 2007). In our 96-well plate assay, no GFP expression was visible in the chosen concentrations under the conditions used.

\section{Plant infections reveal defined expression of TRI5.}

Our main goal was to gain insight into the expression pattern of the trichothecene biosynthesis of $F$. graminearum during the infection process. We created a reporter strain with wild-type behavior on the plant but exhibiting constitutive $d s R e d$ expression as well as GFP expression driven by the endogenous TRI5 promoter. This mutant enables us to pinpoint the expression of TRI5 and the induction of the mycotoxin pathway on the plant under natural growing conditions in real time with cellular resolution. Wheat florets of cv. Nandu were inoculated with $10 \mu \mathrm{l}$ of a conidia suspension $\left(2 \times 10^{4}\right.$ conidia/ml $)$ right after anthesis directly into the floret (Maier et al. 2006). Development of Fusarium head blight (FHB) symptoms was examined for a total of 3 weeks. Infected wheat spikelets were harvested at different time points during that period and sections were examined for $d s R e d$ and GFP expression by fluorescence microscopy. Interestingly, the anthers, that are described as attracting Fusarium spp. to the spikelets and could act like a beacon to the hyphae seeking penetration points (Strange and Smith 1971), were heavily colonized. A lot of aerial mycelium was growing on the surface of the anthers but no GFP fluorescence was visible. In contrast to the anthers, the developing

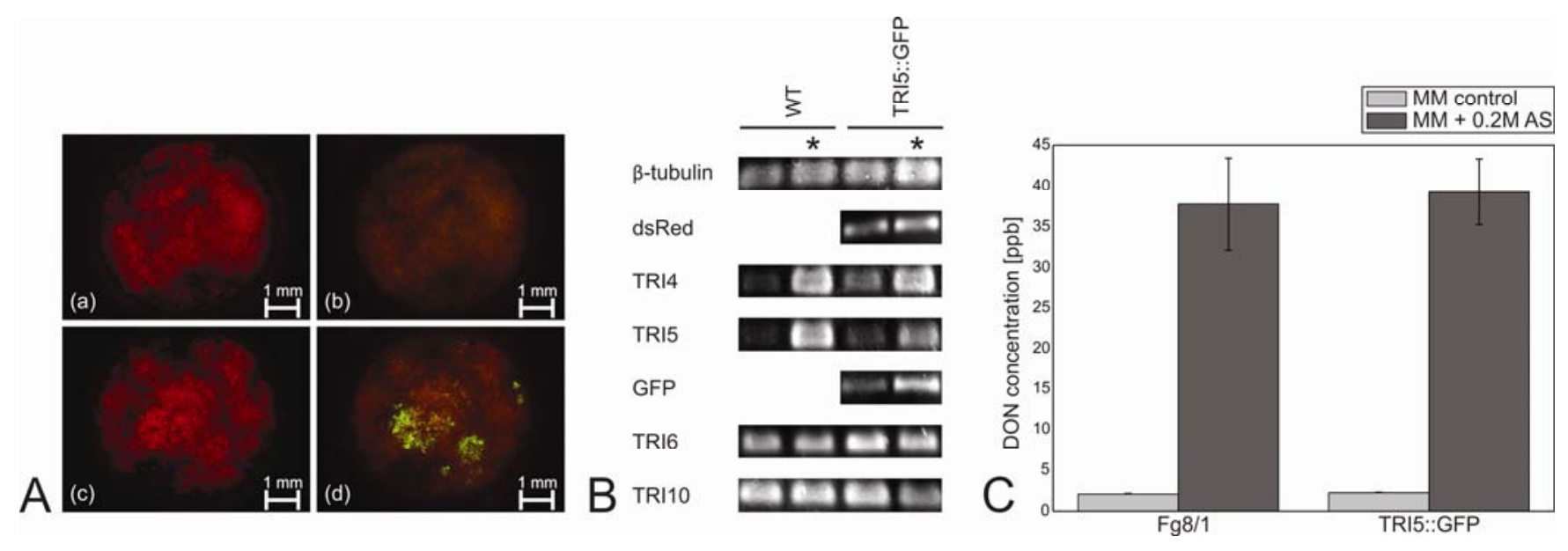

Fig. 4. Results from induction tests with $0.2 \mathrm{M}$ ammonium sulfate (AS) in 96-well plate cultures 4 days after inoculation. A, Fluorescence pictures (a through d) showing representative cultures of a 96-well plate assay with $0.2 \mathrm{M}$ AS as inductive compound; noninduced control culture in $d s R e d$ (a) and green fluorescent protein (GFP) (b) fluorescence; induced culture in dsRed (c) and GFP (d) fluorescence. Interestingly, only defined parts of the hyphal mat show GFP fluorescence (d). B, Gel electrophoresis of reverse-transcriptase polymerase chain reaction (PCR) products from cDNA of wild-type Fusarium graminearum and TRI5prom::GFP samples grown under noninducing and inducing conditions with $0.2 \mathrm{M}$ AS (standard PCR conditions, 30 PCR cycles). Bands marked with an asterisk show PCR product of the specific primer with cDNA from a fungal culture under inducing conditions. $\mathbf{C}$, Representative measurement of DON concentration in parts per billion of wild-type F. graminearum (Fg8/1) and TRI5prom::GFP. Data showing the mean value of 12 repetitions with eight pooled samples; error bars represent the standard error over these samples. Measurements were conducted with the RIDASCREEN DON immunoassay (R-Biopharm AG, Darmstadt, Germany).

Table 1. Chemicals tested for their ability to induce TRI5 under in vitro conditions

\begin{tabular}{lc}
\hline Chemicals tested & Endconcentration in minimal medium \\
\hline Ammonium sulfate & $1 \mathrm{mM}$ to $0.5 \mathrm{M}$ \\
3-Phenylpro-2-enoic acid (cinnamic acid) ${ }^{\mathrm{a}}$ & 0.1 to $5 \mathrm{mM}$ \\
3,4-Dihydroxycinnamic acid (caffeic acid) & 0.1 to $5 \mathrm{mM}$ \\
3-Methoxy-4-hydroxycinnamic acid (ferulic acid) ${ }^{\mathrm{a}}$ & 0.1 to $5 \mathrm{mM}$ \\
Hydrogen superoxide & 0.1 to $1 \mathrm{mM}$ \\
\hline
\end{tabular}

${ }^{a}$ In the case of the phenyl-propanoid acids, $100 \times$ stock solutions were prepared by dissolving in ethanol (96\%, p.A.). Prior to loading into the wells, the appropriate amount of stock solution was added to minimal medium to gain the desired end concentration. Controls consisted of the same amount of ethanol without active compound. 
kernel showed induction of TRI5 already in the early stages of infection. A transsection of a TRI5prom::GFP-infected spikelet of the wheat cv. Nandu is shown in Figure 5B, C, and D. At 3 dai, the mycelium had colonized the developing kernel (Fig. 5C) and trichothecene production was stimulated in this tissue, as indicated by GFP expression (Fig. 5D). Additionally, there was a weak $G F P$ induction of individual hyphae growing adaxially on the palea and the lemma. However, the majority of hyphae colonizing these floral structures remained uninduced. At 4 dai, the mutant had colonized the complete tissue from the point of inoculation to the basal zone of the spikelet. Here, the vascular vessels of the rachis divided in the rachis node and formed the rachilla. The vessels leading to the inoculated floret were completely colonized, as indicated by $d s R e d$ fluorescence (Fig. 5F). This tissue was not only completely colonized but also induced $G F P$ fluorescence strongly and uniformly, indicating a dramatic induction of the trichothecene pathway (Fig. 5G and $\mathrm{H}$ ). During the course of infection, the fungus starts to spread through the rachis over the complete spike, and very similar situations can be observed when myce-
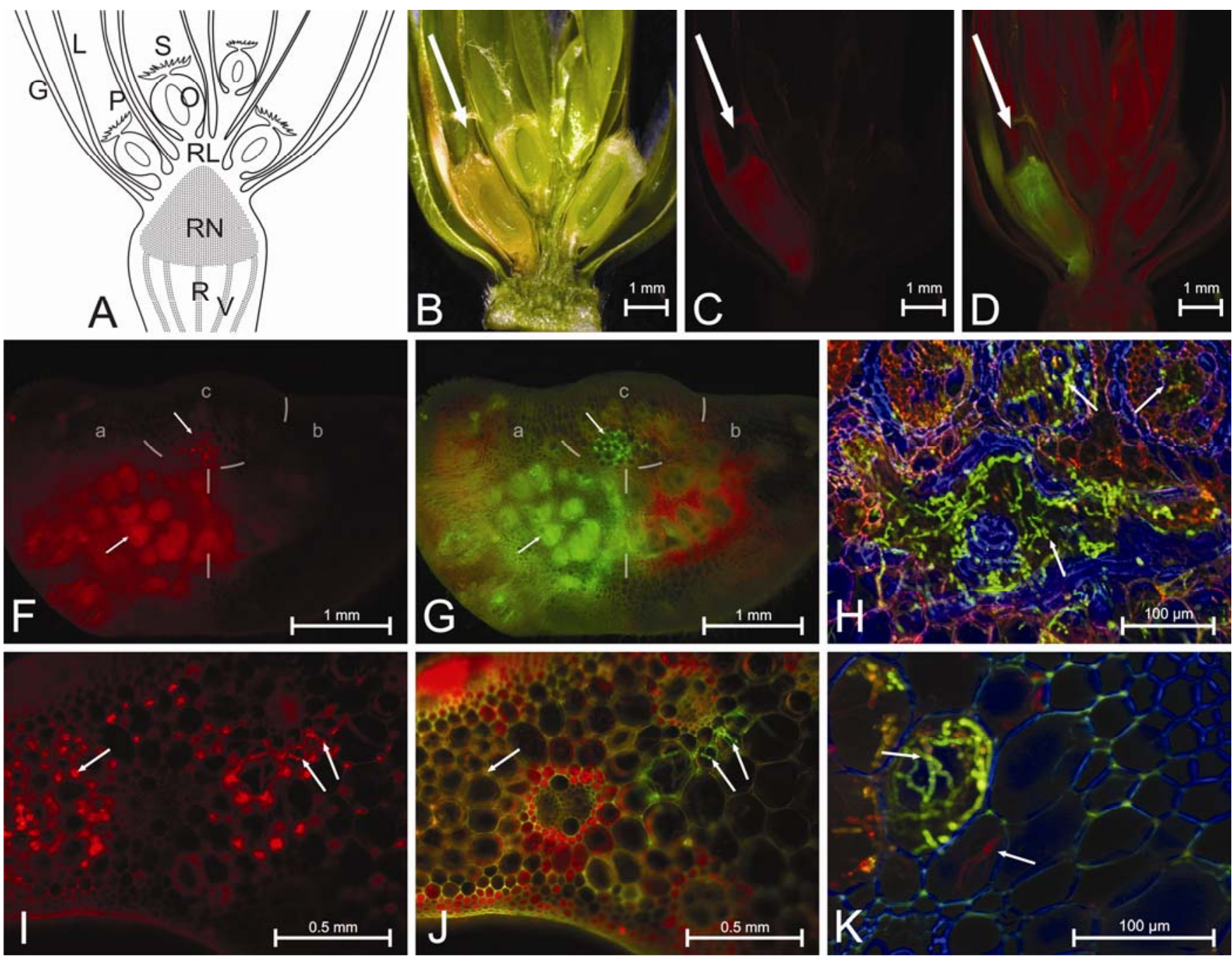

Fig. 5. Fluorescence pictures of TRI5prom::GFP-infected wheat spikes (cv. Nandu), dissected and microscopically examined after different time points. A, Schematic drawing of a wheat spikelet, modified after Kang and Buchenauer (2000), showing glume (G), lemma (L), palea (P), stigma (S), ovary (O), rachilla (RL), rachis node (RN), rachis (R), and vascular vessels (V). B through D, Transsection of a TRI5prom::GFP-infected spikelet at 3 days after inoculation (dai) (arrows mark point of infection). B, In the brightfield, browning and bleaching of the infected flower can be observed. C, Examination under $d s R e d$-exciting conditions reveals the complete colonization of the developing kernel. Simultaneously, a high expression of green fluorescent protein (GFP) takes place (D, GFP exciting blue light). Photos were taken with the Leica MZ FL III fluorescence microscope. F through $\mathbf{H}$, Top view of a cross section through the rachis node of the inoculated spikelet at 4 dai: a, inoculated floret of the spikelet; b, adjacent, not inoculated flower of the spikelet; $\mathrm{c}$, rachis leading to upper and lower spikelet. F, The reporter strain has colonized the complete tissue around the vascular vessels supplying the inoculated flower of the spikelet (a, lower arrow on the left side) (dsRed exciting conditions). Additionally, mycelium is growing in the apoplast between the rachis parenchymal cells (c, upper arrow). G, The complete mycelium growing in the rachis node as well as in the rachis of the inoculated floret is expressing GFP, indicating a massive induction of the trichothecene biosynthesis pathway in this tissue (GFP exciting conditions). Photos were taken with the Leica MZ FL III fluorescence microscope. H, Magnified view of the central region of the rachis node after 4 days of infection. The tissues between the vascular vessels (lower arrow in the middle) as well as some of the vascular vessels (upper arrows) are completely colonized. Overlay image of photos taken with the 4',6-diamidino-2-phenyliadole (DAPI), dsRed, and GFP filters individually and combined with the Zeiss AxioVision software. Image was taken with a Zeiss AxioImager.Z1 fluorescence microscope. I through $\mathbf{K}$, Cross sections of a rachis part adjacent to the inoculated spikelet at 5 dai. The rachis is completely colonized by the mutant. The majority of the hyphae is growing in the apoplast between the parenchymal cells of the rachis (I, arrow on left), where GFP fluorescence was not observed ( $\mathrm{J}$, arrow on the left). Induction of GFP can be found where the mutant grows intracellularly (I and $\mathrm{J}$, arrows on the right) in the parenchymal cells. Photos were taken with the Leica MZ FL III fluorescence microscope. K, Higher magnification of a rachis cross section at 5 dai. Most of the mycelium growing intracellularly is expressing GFP (upper arrow) but there are also hyphae growing inside of the cells that only show $d s R e d$ (lower arrow). Overlay image of photos taken with the DAPI, dsRed, and GFP filters individually and combined with the Zeiss AxioVision software. Image was taken with a Zeiss AxioImager.Z1 fluorescence microscope. 
lium starts to grow into the rachis node of remote spikelets. Here, our strain also showed high GFP fluorescence in this area particularly. Although TRI5 induction is majorly seen in the rachis node, the parenchymal cells of the rachis, which act as infection pathways to adjacent spikelets, are in large part free of GFP. In rachis cross sections of infected plants (5 dai) we found $G F P$-expressing mycelium adjacent to hyphae that did not show GFP fluorescence (Fig. 5I through K). Here, the fungus was predominantly growing in the apoplastic space where the vast majority of mycelium was only expressing $d s R e d$ (Fig. 5I). High GFP fluorescence can be observed when the mycelium manages to break through the cell wall and is growing intracellularly (Fig. 5J and K). At 10 dai, the complete spike was infected and, after that time, hyphae can be found growing in the vascular vessels, particularly the phloem (Fig. 6A). In the extensively infected vascular vessels, GFP fluorescence seems to be restricted to the hyphae growing in the phloem indicating a specific induction in this location. After 22 days of infection, $F$. graminearum had spread all over the spike and mycelium was descending down the stem below the spike. There was an extensive colonization of the complete tissue, including a massive invasion of hyphae into the phloem and xylem (Fig. 6B and C). In spite of the massive fungal growth, no GFP induction was visible during this late stage of plant colonization, except for the last node of the spike where the stem merges into the spike (Fig. 6B). The stem was colonized by hyphae growing on the surface of the inner parenchy-

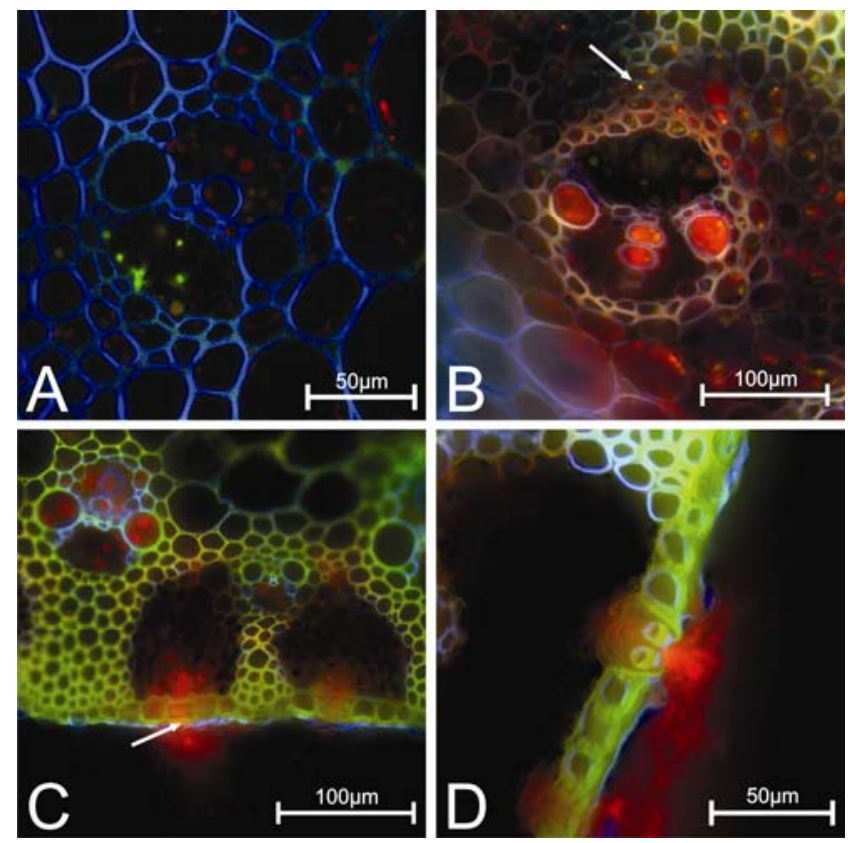

Fig. 6. A, Laser scanning microscopy image of a cross section of the rachis located under the inoculated spikelet 10 days after inoculation. The green fluorescent protein $(G F P)$-enhanced fungus has colonized the tissue completely and grows in parenchymal cells as well as in the vascular vessels (dsRed fluorescence). TRI5 induction is confined to the hyphae growing in the phloem tissue. Plant cell wall autofluorescence was excited with $405 \mathrm{~nm}$ to distinguish between autofluorescence and fluorescence signals of GFP and $d s R e d$. Photo was taken with a Zeiss LSM 710. B through D, Fluorescence microscopy images of transsections of the wheat stem after 22 days of infection. B, The reporter strain has completely colonized the vascular vessels of the last node at the bottom of the spike where the spike meets the stem; isolated hyphae are showing GFP expression (arrow). C, Section of the wheat stem, approximately $5 \mathrm{~cm}$ under the spike. The vascular vessels are heavily infected and hyphae are growing through the stomata to the surface of the stem (arrow). All hyphae are expressing dsRed but no GFP fluorescence is visible. $\mathbf{D}$, Hyphae breaking through stomata reaching the surface of the wheat stem. Photos were taken with a Zeiss AxioImager.Z1. mal cells forming the cavity of the stem. Examination of cross sections of the stem located approximately $5 \mathrm{~cm}$ under the last nodium of the spike showed mycelium using the stomata as gateways to grow to the surface of the stem (Fig. 6D). Interestingly, no GFP fluorescence was detectable in the infected cells of the stem, not even at the growth front.

\section{GFP expression resembles TRI5 expression in planta.}

To prove whether or not the GFP fluorescence observed during plant infection reflects an increased expression of TRI5, we had a closer look at the expression levels of these genes under infection conditions. The results from our expression studies show a higher expression of TRI5 in the rachis node compared with the expression levels in the adjacent rachis tissue (Fig. 7). The same expression pattern can be found for the abundance of $G F P$ transcript.

\section{DISCUSSION}

Consumption of mycotoxin-contaminated cereals and their products can lead to health-threatening toxication in humans and animals. F. graminearum infections on cereals and corn lead to heavy contamination with mycotoxins, especially trichothecenes. Biosynthesis of these mycotoxins is not constitutively active but specifically induced during the time course of colonization of the host plant tissue. Therefore, it is necessary to understand the mechanisms that lead to an induction of the trichothecene pathway during the course of infection. Although the genes of the trichothecene gene cluster are known and regulatory elements are described, little is known about the factors that influence regulation of this pathway on the plant. We present one way to gain further knowledge of the host-pathogen relationship between $F$. graminearum and Triticum aestivum (common wheat). We created a mutant exhibiting $d s R e d$ driven by the constitutive $g p d A$ promoter and GFP driven by the endogenous promoter of TRIS, the gene coding for the key enzyme of the trichothecene pathway.

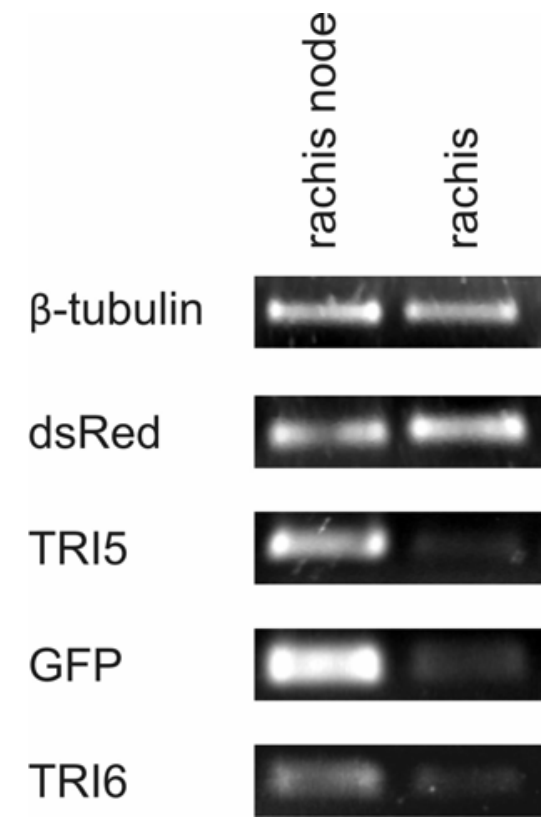

Fig. 7. Gel-electrophoresis of reverse-transcriptase polymerase chain reaction (PCR) products with cDNA isolated from infected plant tissue after 4 days of infection (standard PCR conditions, 32 PCR cycles). The expression of TRI5 and green fluorescent protein $(G F P)$ in the rachis node is upregulated compared with the expression level of these genes in the adjacent rachis. The expression pattern of TRI5 resembles the expression pattern of $G F P$. 
The reporter strain shows wild-type-like behavior.

The creation of the mutant by homologous integration into the TRI5 locus is a crucial step. It is necessary to maintain the wild-type-like character of the $G F P$-enhanced strain to draw conclusions about the behavior of the wild type itself. In plant infection assays on wheat cv. Nandu plants, the reporter strain and the wild type showed the same infection behavior and inoculated plants developed typical FHB disease symptoms after 3 weeks of inoculation (Fig. 3). Several binding sites for TRI6, a zinc finger protein involved in the regulation of the trichothecene pathway, can be found in the promoter region of TRI5 and other TRI cluster genes (Proctor et al. 1995b). Recent findings show that an integration of a transformation vector can alter the pathway gene expression and lead to a higher production of 15-acetyldeoxynivalenol (Chen et al. 2000). Therefore, we examined the production of DON as well as the expression patterns of TRI5, TRI4, TRI6, and TRI10 in both the wild-type and the reporter strain. The genes TRI5 and TRI4 are the first two genes of the trichothecene pathway, with the trichodiene synthase (TRI5) as the key enzyme and TRI4, coding for a cytochrome P450 monooxygenase, catalyzing the subsequent steps after formation of trichodiene (Tokai et al. 2007). With TRI6 and TRI10, we chose two genes that are described to be regulators of the TRI5 gene cluster (Kimura et al. 2007). The levels of gene expression of all tested genes were very similar between the wild type and TRI5prom::GFP, suggesting that the integration of the transformation vector did not lead to an altered expression of TRI5. However, there was a clear-up regulation of TRI5 and TRI4 in mycelium growing in MM supplemented with $0.2 \mathrm{M}$ AS (Fig. 4B). Either the transcript levels of TRI6 and TRIIO are stable under the chosen conditions or changes in expression level are too small to be detected in our RT-PCR. The increased expression of TRI5 comes along with an increased level of GFP transcripts, revealing the direct relationship between observed GFP fluorescence and TRI5 gene expression (Fig. 4B). The raised gene expression is in direct relationship to the increased concentration of DON in the medium (Fig. 4C). Both the wild type and the reporter strain produce very small amounts of DON in pure $\mathrm{MM}$, whereas toxin concentration is clearly elevated in MM with added AS (Fig. 4C). We succeeded in the creation of the $T R I 5$ prom::GFP reporter strain as well as in the preservation of its wild-type-like character. The expression patterns of the tested trichothecene cluster genes in the wild type and in the $G F P$ reporter strain are the same in inducibility and in expression levels (Fig. 4B), proving that the integration of the TRI5 promoter construct into the locus did not alter the expression of the targeted genomic region. In addition to that, the expression of GFP reports the expression of TRI5 and correlates with the microscopically observed GFP fluorescence. TRI5 expression is in direct relationship to DON production as well. Again, the wild type and the GFP strain behaved very similar under inducing and noninducing conditions, with an approximately 18 -fold higher DON production under the influence of AS (Fig. 4C).

\section{Induction test with bioimaging.}

We developed an in vitro test system with minicultures in 96-well plates to compare wild-type Fusarium spp. with the TRI5prom::GFP strain. This system can easily be used to test agronomically interesting compounds such as nutrients or pesticides for their effects on trichothecene production. Together with our reporter strain, the test system provides a fast and inexpensive way to screen for possible inducers or repressors of the trichothecene pathway by simple bioimaging. Most other systems rely on HPLC-MS or LC/MS measurements that are time consuming and elaborate (Bily et al. 2004; Evans et al. 2000). In addition to AS, we tested three phenolic compounds found in plant cell walls as well as hydrogen superoxide for their ability to induce TRI5 expression. Accumulation of lignin and plant phenolic acids in cell walls during HR may contribute to resistance to Fusarium spp. (Menden et al. 2007). These compounds seem to have an influence on disease severity and resistance of maize against $F$. graminearum infection (Bakan et al. 2003; Santiago et al. 2007). The most abundant phenolic acid found in plant cell walls is ferulic acid. It contributes to stability of the cells as well as to the autofluorescence shown by plant tissue (Klepacka and Fornal 2006). Interestingly, the abundance of ferulic acid in the wheat plant cell wall and resistance to seedborne $F$. graminearum infection increases when wheat plants are treated with chitosan, a polysaccharide also found in fungal cell walls (Reddy et al. 1999). The tested phenolic acids did not induce TRI5 expression but they severely inhibited fungal growth and germination at a concentration higher than $1 \mathrm{mM}$. Other common compounds found in pathogen-activated $\mathrm{HR}$ are reactive oxygen species such as hydrogen superoxide $\left(\mathrm{H}_{2} \mathrm{O}_{2}\right)$ (Shetty et al. 2008). Hydrogen superoxide and other reactive oxygen species-developing compounds were recently described to have a positive impact on toxin production in liquid culture, even that a certain amount of $\mathrm{H}_{2} \mathrm{O}_{2}$ has to be present in the fungal environment to ensure trichothecene production (Ponts et al. 2006, 2007). We tested several concentrations of $\mathrm{H}_{2} \mathrm{O}_{2}$ in MM for TRI5 induction but GFP fluorescence was not observed. It has to be stated that we chose MM for our induction experiments because it is a very basic yet defined liquid growth medium consisting of only minerals and glucose, and shows no autofluorescence in the wavelengths used for microscopic examination. The commonly used complete medium (CM) GYEP, that slightly induces TRI5 by itself, shows a very high fluorescence in the GFP emission bandwidth that cloaks GFP signals and is, therefore, unsuitable for fluorescence microscopy. Nevertheless, we have hints suggesting that TRI5 induction behavior depends on the state of nutrition of $F$. graminearum (data not shown). The GFP system allows us to detect differences in gene expression within a uniformly grown fungal colony. Interestingly, only parts of the colony express GFP even under inducing nitrogen oversupply (Fig. 4A). Our results seem to indicate that several factors are required to induce TRI5 expression and, thus, DON production. In this case, nitrogen supply alone is not sufficient to give a uniform induction. Because GFP induction was only seen in aerial mycelium grown out of the otherwise submerged fungal mat, oxygen supply may play a supporting role. The complex nature of in vitro TRIS induction is supported by other results, too. Under our test conditions, we could not detect that hydrogen superoxide stress leads to TRI5 induction. We conclude that special growth conditions plus an inducible substance such as nitrogen, $\mathrm{H}_{2} \mathrm{O}_{2}$, or possibly others are needed to induce TRI5 in vitro. Whether this is of relevance to the conditions needed for induction during plant infection remains to be tested.

\section{Plant infections reveal tissue-specific TRI5 induction.}

Therefore, our main goal was to develop a mutant that enables us to follow the infection and induction of the trichothecene biosynthesis pathway of $F$. graminearum in real time and on a cellular level. The ubiquitous expression of $d s R e d$ eases the identification of the fungus during histological examinations. It also assists the separation of the natural autofluorescence occurring in plants from the valuable GFP and $d s R e d$ signals of the fungus. We can show, for the first time, that TRIS expression is not ubiquitous during the course of infection but seems to be affected and specifically induced by the plant tissue through which the hyphae are growing. We demonstrated 
recently that Fusarium spp. infect the plant by colonizing the developing kernel and that DON is not a virulence factor during infection through the fruit coat (Jansen et al. 2005). Our TRI5prom::GFP strain reveals that the developing kernel has influence on the induction of trichothecenes (Fig. 5D); indeed, this tissue massively induces fungal GFP fluorescence. Occasionally, comparably weak GPF induction was detected on the adaxial side of the flower leaves. This is in occurrence with results that demonstrate DON production in very early stages of spikelet infection (Evans et al. 2000). Surprisingly, the anthers, which are believed to attract the hyphae to grow directly to the floret (Strange and Smith 1971), did not show induction of TRI5, although they were heavily overgrown. In later stages of approximately 4 to 7 days, the rachis node between the rachilla and the rachis was the point where the most extensive GFP fluorescence was found (Fig. 5F through H). RT-PCR experiments with isolated material from infected rachis nodes showed a high expression of TRI5 as well as GFP and a much lower expression of these genes in the adjacent rachis (Fig. 7). These results showed clearly that the observed GFP fluorescence concurred in TRI5 expression not only in vitro but also during the course of infection in planta. The formation of heavy cell wall thickenings in this area prevents the trichothecene-deficient mutant $\Delta$ tri5 from growing into the rachis and from colonizing the rest of the spike (Maier et al. 2006). Interestingly, plant infection assays with $\Delta f g l l$ lacking a gene for a secreted lipase of $F$. graminearum show that this mutant is also impaired in the ability to overcome the rachis node and spread through the entire spike (Voigt et al. 2005). A barrier is formed that seals the $\Delta f g l l$ mutant in the inoculated floret and prevents colonization of adjacent tissue. Consequently, both virulence factors, FGL1 as well as TRI5, are induced during infection of the plant and both of them have to be present for an effective colonization of wheat. Toxin measurements of $\Delta f g l l$-infected spikelets revealed an even higher concentration of DON than spikelets inoculated with the wild type (Voigt et al. 2007). However, the $\Delta f g l l$ mutant was still trapped and the spikelet was sealed from the rest of the tissue. Therefore, we conclude that it is unlikely that the same plant defense mechanism leads to the confinement of the $\Delta f g l l$ and $\Delta T R I 5$ in the inoculated spikelet. Our results stress the overwhelming importance of the rachis node of wheat plants for their defense against $F$. graminearum infections. We demonstrate that the GFP reporter strain indicates a massive induction of the trichothecene biosynthesis pathway, especially when it reaches this tissue, suggesting that trichothecenes are needed to suppress plant defense and prevent the plant from sealing the inoculated tissue.

The question of whether trichothecenes are necessary for a successful infection of maize cobs is still to be answered. Results of field trials conducted by Harris and associates (1999) seem to indicate that trichothecenes contribute to the colonization of maize ears whereas Maier and associates (2006) could show that these mycotoxins are not required for a successful infection of maize under their conditions. The fact that $F$. graminearum produces high amounts of trichothecenes on maize, although these mycotoxins seem to be of minor importance for successful infection, raises the question of whether there is a common compound or physiological condition in wheat and maize that triggers TRI5 induction, regardless of whether or not the invading fungus needs DON for successful colonization.

Our GFP-enhanced strain can help to understand the physiological role trichothecenes play during the course of infection. For the first time, it is possible to monitor initiation of trichothecene production under infection conditions on the plant as well as in vitro. On the plant, the TRI5prom::GFP strain reveals that TRI5 expression is tightly regulated during wheat infection with the highest induction in the transition zone of the rachis node, where the rachilla and the rachis divide. This section of the spikelet seems to be of major importance for the resistance of wheat against $F$. graminearum. Furthermore, the reporter strain now allows us to screen for possible inducers or repressors of the trichothecene pathway and could provide a new tool to diminish mycotoxin burden in cereals.

\section{MATERIALS AND METHODS}

\section{Strains and cultivars.}

The $F$. graminearum isolate $F g$ 8/1 (Miedaner et al. 2000) was used to clone the $G F P$-enhanced strain. Wheat cv. Nandu (Lochow-Petkus, Bergen-Wohlde, Germany) was used for all inoculation experiments as host plant.

\section{Plasmid construction.}

Plasmids pIGPAPA (Lee et al. 2003), pGEM-T (Promega, Mannheim, Germany), pAN7-1 (Punt et al. 1987), pPgpddsRed (Mikkelsen et al. 2003), and pII99 (Namiki et al. 2001) were modified and used in this study.

\section{Chemicals and media.}

MM and CM, according to Leach and associates (1982), were used as liquid media or solidified with $1.5 \%$ agar. Conidiation was performed on SNA medium according to Nirenberg (1981). All DNA-modifying enzymes were retrieved from MBI Fermentas (St. Leon-Rot, Germany).

\section{Fungal transformation, plant inoculation, and growth conditions.}

Fungal transformation, induction of conidiation, wheat plant growth conditions, and spikelet inoculation were performed according to Maier and associates (2006).

\section{Standard molecular techniques.}

Extraction of genomic DNA, Southern blot analysis, RNA extraction, and RT-PCR (Table 2, primer sequences) was performed according to Maier and associates (2006). All PCR reactions were carried out in a total volume of $25 \mu \mathrm{l}$ in a MWG Primus PCR cycler. The standard PCR mix consisted of $0.1 \mathrm{U}$ of 5-PRIME Taq polymerase, $2.5 \mu$ of $10 \times$ Taq polymerase buffer, $0.625 \mu \mathrm{l}$ of each primer ( $10 \mathrm{pmol}$ of stock), $0.625 \mu \mathrm{l}$ of $10 \mathrm{mM}$ dNTP mix, a variable volume of template DNA, and doubledistilled $\mathrm{H}_{2} \mathrm{O}$ up to $25 \mu \mathrm{l}$ of end volume. The standard PCR program was composed of 4 min of initial denaturation at $95^{\circ} \mathrm{C}$ followed by 30 cycles of $30 \mathrm{~s}$ of denaturation, $30 \mathrm{~s}$ of annealing, and $30 \mathrm{~s}$ of elongation. The program ended with a 10-min final elongation and storage of the PCR products at $4^{\circ} \mathrm{C}$. Fusion PCR was performed according to Amberg and associates (1995). Digoxigenin (DIG) labeling of probes for Southern blot characterization was done by standard PCR with genomic DNA as template and primers TRI5fwd/rev and dsRedfwd/rev (Table 2) under the presence of DIG-dUTP as recommended by the manufacturer (Roche, Mannheim, Germany). After blotting, band sizes were estimated by comparison with the DIG-labeled DNA markers DIGIII and DIGVII (Roche). Genomic DNA and cDNA sequences for primer design of the TRI5 gene cluster genes were derived from the $F$. graminearum sequence databank of the BROAD Institute.

\section{Monitoring TRI5 induction and DON production under in vitro and in planta conditions.}

Induction assays were carried out in 96-well plates (Microtest 96-Well Assay Plate, black, flat bottom, TC surface, sterile) (BD Falcon, Le Pont De Claix, France) with MM as substrate. Each well was loaded with 100 liters of medium and inoculated with 10 liters of a conidia suspension $\left(2 \times 10^{4}\right.$ conidia/ml $)$. 
Table 2. Primer sequences used for cloning, reverse-transcriptase polymerase chain reaction, and labeling of hybridization probes as well as their corresponding fragment sizes

\begin{tabular}{|c|c|c|}
\hline Name & Sequence & $\begin{array}{c}\text { Fragment } \\
\text { size (bp) }\end{array}$ \\
\hline TRISProm-fwd & GTACTCTGTACAGAGTACTTCGTCGA & 1,095 \\
\hline TRI5Prom-rev & AGGCTGAGCAAAGTGGTGGGGAG & $\ldots$ \\
\hline TRI5-GFP-fwd & TTTGCACTATGCTTACAACAAGGCTGCCCACCACTTTGCTCAGCCTGTGAGCAAGGGCGAGGAGCTGTTCAC & $\ldots$ \\
\hline GFP-HindIII-rev & CCCAAGCTTGGGGGTCGACGGTATCGATAAGA ${ }^{\mathrm{b}}$ & $\ldots$ \\
\hline TRI5-fwd & ATGACTACCCTCAATTCCTTCGT & 451 \\
\hline TRI5-rev & GAACTTCTTGGCGTCCTCTG & $\ldots$ \\
\hline TRI4-fwd & GTACCGTTATCCTGTTTGCTG & 464 \\
\hline TRI4-rev & CAAAGGCCATAGTGTATCCGA & $\ldots$ \\
\hline TRI6-fwd & AGACTTTGTACTCCGAAGAACCA & 382 \\
\hline TRI6-rev & TTGTCCTTCCTTGTCTTGCCA & $\ldots$ \\
\hline TRI10-fwd & GACCTCATCACTCAGATTTACGCC & 306 \\
\hline TRIIO-rev & СТСТTСТСТTCCAAGCGTCTTCC & $\ldots$ \\
\hline GFP2-fwd & TCTTCAAGTCCGCCATGCCC & 453 \\
\hline GFP2-rev & TCCATGCCGTGAGTGATCCC & $\ldots$ \\
\hline dsRed-fwd & CGAGGACGTCATCAAGGAGT & 366 \\
\hline$d s R e d$-rev & GCCGATGAACTTCACCTTGT & $\ldots$ \\
\hline Betatub-fwd & CCCAACAACGTCCAAACTTCC & 195 \\
\hline Betatub-rev & CCTCGGTGAATTCCATCTCGTC & $\ldots$ \\
\hline
\end{tabular}

a Italics: 48-bp homology to TRI5 fragment.

${ }^{\mathrm{b}}$ Italics: HindIII recognition sequence.

Different chemicals were tested for their ability to induce TRI5 under in vitro conditions (Table 2, compound and concentration). In the case of added AS, the required amount for a $0.2-\mathrm{M}$ concentration was dissolved in $\mathrm{MM}$ and sterile filtrated prior to loading into the wells. Controls consisted of MM with the same amount of solvent or pure medium. All 96-well plates were placed in moistened plastic bags to prevent drying while incubating at $28^{\circ} \mathrm{C}$ in the dark. The quality of the $G F P$ and dsRed signals over time was examined with the epifluorescence microscopes described below. For gene expression analysis, RNA was extracted from pooled mycelium of a 96well plate and plant tissue (rachis node and adjacent rachis) at 4 dai. DON concentration was measured in the remaining medium after the harvest of the mycelium. For statistical analysis, eight individual wells were pooled, thus creating 12 samples out of one 96-well plate, and the experiment was repeated four times. Concentration of produced DON was estimated using the RIDASCREEN DON enzymatic immunoassay.

\section{Histological examination of TRI5 induction during plant infection.}

Spikelets and rachis of infected wheat spikes between 3 and 7 dai were hand sectioned and monitored by fluorescence microscopy. Fungal infection was determined by following the constitutively expressed $d s R e d$ signal. Additionally, the GFP signal derived from the TRI5 promoter GFP fusion was recorded. Co-localization of both reporter proteins enabled us to assign trichothecene-inducing conditions to specific plant tissues and even cells. Fluorescence microscopy was carried with a Leica MZ FL III microscope equipped with a Leica DFC 500 fluorescence camera, a Zeiss Axio Imager.Z1 microscope equipped with a AxioCam MRm fluorescence camera and a Zeiss LSM 710 laser scanning microscope.

\section{ACKNOWLEDGMENTS}

We thank B. Doormann for her assistance in preparing the manuscript.

\section{LITERATURE CITED}

Amberg, D. C., Botstein, D., and Beasley, E. M. 1995. Precise gene disruption in Saccharomyces cerevisiae by double fusion polymerase chain reaction. Yeast 11:1275-1280.

Bakan, B., Bily, A. C., Melcion, D., Cahagnier, B., Regnault-Roger, C.,
Philogène, B. J. R., and Richard-Molard, D. 2003. Possible role of plant phenolics in the production of trichothecenes by Fusarium graminearum strains on different fractions of maize kernels. J. Agric. Food Chem. 51:2826-2831.

Bily, A. C., Reid, L. M., Savard, M. E., Reddy, R., Blackwell, B. A. Campbell, C. M., Krantis, A., Durst, T., Philogène, B. J. R., Arnason, J. T., and Regnault-Roger, C. 2004. Analysis of Fusarium graminearum mycotoxins in different biological matrices by LC/MS. Mycopathologia 157:117-126.

Chen, L., McCormick, S. P., and Hohn, T. M. 2000. Altered regulation of 15-acetyldeoxynivalenol production in Fusarium graminearum. Appl. Environ. Microbiol. 66:2062-2065.

Covarelli, L., Turner, A. S., and Nicholson, P. 2004. Repression of deoxynivalenol accumulation and expression of TRI genes in Fusarium culmorum by fungicides in vitro. Plant Pathol. 53:22-28.

Desjardins, A. E., Hohn, T. M., and McCormick, S. P. 1993. Trichothecene biosynthesis in Fusarium species: Chemistry, genetics and significance. Microbiol. Rev. 57:595-604.

Desjardins, A. E., Proctor, R. H., Bai, G., McCormick, S. P., Shaner, G., Buechley, G., and Hohn, T. M. 1996. Reduced virulence of trichothecene nonproducing mutants of Gibberella zeae in wheat field tests. Mol. Plant-Microbe Interact. 9:775-874.

Evans, C. K., Xie, W., Dill-Macky, R., and Mirocha, C. J. 2000. Biosynthesis of deoxynivalenol in spikelets of barley inoculated with macroconidia of Fusarium graminearum. Plant Dis. 84:654-660.

Goswami, R. S., and Kistler, C. H. 2004. Heading for disaster: Fusarium graminearum on cereal crops. Mol. Plant. Pathol. 5:515-525.

Harris, L. J., Desjardins, A. E., Plattner, R. D., Nicholson, P., Butler, G., Young, J. C., Weston, G., Proctor, R. H., and Hohn, T. M. 1999. Possible role of trichothecene mycotoxins in virulence of Fusarium graminearum on maize. Plant Dis. 83:954-960.

Hohn, T. M., and Desjardins, A. E. 1992. Isolation and gene disruption of the Tox5 gene encoding trichodiene synthase in Gibberella pulicaris. Mol. Plant-Microbe Interact. 5:249-156.

Jansen, C., von Wettstein, D., Schäfer, W., Kogel, K. H., Felk, A., and Maier, F. J. 2005. Infection patterns in barley and wheat spikes inoculated with wild-type and trichodiene synthase disrupted Fusarium graminearum. PNAS 46:16892-16897.

Kang, Z., and Buchenauer, H. 2000. Ultrastructural and immunocytochemical investigation of pathogen development and host responses in resistant and susceptible wheat spikes infected by Fusarium culmorum. Physiol. Mol. Plant Pathol. 57:255-268.

Kimura, M., Tokai, T., Takahashi-Ando, N., Ohsato, S., and Fujimura, M. 2007. Molecular and genetic studies of Fusarium trichothecene biosynthesis: Pathways, genes, and evolution. Biosci. Biotechnol. Biochem. 71:2105-2123.

Klepacka, J., and Fornal, L. 2006. Ferulic acid and its position among the phenolic compounds of wheat. Crit. Rev. Food Sci. Nutr. 46:639-647.

Leach, J., Lang, B. R., and Yoder, O. C. 1982. Methods for selection of mutants and in vitro culture of Cochliobolus heterostrophus. J. Gen. Microbiol. 128:1719-1729.

Lee, J., Lee, T., Lee, Y.-W., Yun, S.-H., and Turgeon, G. B. 2003. Shifting 
fungal reproductive mode by manipulation of mating type genes: Obligatory heterothallism of Gibberella zeae. Mol. Microbiol. 50:145-152.

Lemmens, M., Haim, K., Lew, H., and Ruckenbauer, P. 2004. The effect of nitrogen fertilization on Fusarium Head Blight development and deoxynivalenol contamination in wheat. J. Phytopathol. 152:1-8.

Maier, F. J., Miedaner, T., Hadeler, B., Felk, A., Salomon, S., Lemmens, M., Kassner, H., and Schäfer, W. 2006. Involvement of trichothecenes in fusarioses of wheat, barley and maize evaluated by gene disruption of the trichodiene synthase (TRI5) gene in three field isolates of different chemotype and virulence. Mol. Plant Pathol. 7:449-461.

Marasas, M. F. O., Nelson, P. E., and Toussoun, T. A. 1984. Toxigenic Fusarium Species: Identity and Mycotoxicology. Pennsylvania State University Press, University Park, U.S.A.

Menden, B., Kohlhoff, M., and Moerschbacher, B. M. 2007. Wheat cells accumulate a syringyl-rich lignin during the hypersensitive resistance response. Phytochemistry 68:513-520.

Miedaner, T., Reinbrecht, C., and Schilling, A. G. 2000. Association among aggressiveness, fungal colonization, and mycotoxin production of 26 isolates of Fusarium graminearum in winter rye head blight. J. Plant Dis. Prot. 107:124-134.

Mikkelsen, L., Sarrocco, S., Lübeck, M., and Jensen, D. F. 2003. Expression of the red fluorescent protein DsRed-Express in filamentous ascomycete fungi. FEMS (Fed. Eur. Microbiol. Soc.) Microbiol. Lett. 223:135-139.

Namiki, F., Matsunaga, M., Okuda, M., Inoue, I., Nishi, K., Fujita, Y., and Tsuge, T. 2001. Mutation of an arginine biosynthesis gene causes reduced pathogenicity in Fusarium oxysporum f. sp. melonis. Mol. PlantMicrobe Interact. 14:580-584.

Nirenberg, H. 1981. A simplified method for identifying Fusarium spp. occurring on wheat. Can. J. Bot. 59:1599-1609.

Ponts, N., Pinson-Gadais, L., Verdal-Bonnin, M., Barreau, C., and Richard-Forget, F. 2006. Accumulation of deoxynivalenol and its 15acetylated form is significantly modulated by oxidative stress in liquid cultures of Fusarium graminearum. FEMS (Fed. Eur. Microbiol. Soc.) Microbiol. Lett. 258:102-107.

Ponts, N., Pinson-Gadais, L., Barreau, C., Richard-Forget, F., and Ouellet, T. 2007. Exogenous $\mathrm{H}_{2} \mathrm{O}_{2}$ and catalase treatments interfere with TRI genes expression in liquid cultures of Fusarium graminearum. FEBS (Fed. Eur. Biochem. Soc.) Lett. 581:443-447.

Proctor, R. H., Hohn, T. M., and McCormick, S. P. 1995a. Reduced virulence of Gibberella zeae caused by disruption of a trichothecene toxin biosynthetic gene. Mol. Plant-Microbe Interact. 8:593-601.

Proctor, R. H., Hohn, T. M., McCormick, S. P., and Desjardins, A. E. 1995b. TRI6 encodes an unusual zinc finger protein involved in regulation of trichothecene biosynthesis in Fusarium sporotrichioides. Appl. Environ. Microbiol. 61:1923-1930.

Punt, P. J., Oliver, R. P., Dingemanse, M. A., Pouwels, P. H., and van den Hondel, C. A. 1987. Transformation of Aspergillus based on the hygromycin B resistance marker from Escherichia coli. Gene 56:117124.

Reddy, M. V. B., Arul, J., Angers, P., and Couture, L. 1999. Chitosan treatment of wheat seeds induces resistance to Fusarium graminearum and improves seed quality. J. Agric. Food Chem. 47:1208-1216.

Rocha, O., Ansari, K., and Doohan, F. M. 2005. Effects of trichothecene mycotoxins on eukaryotic cells: A review. Food Addit. Contam. 22:369378.

Santiago, R., Reid, L. M., Arnason, J. T., Zhu, X., Martinez, N., and Malvar, R. A. 2007. Phenolics in maize genotypes differing in susceptibility to Gibberella stalk rot (Fusarium graminearum Schwabe). J. Agric. Food Chem. 55:5186-5193.

Shetty, N. P., Jørgensen, H. J. L., Jensen, J. D., Collinge, D. B., and Shetty, H. S. 2008. Roles of reactive oxygen species in interactions between plants and pathogens. Eur. J. Plant Pathol. 121:267-280.

Strange, R. N., and Smith, H. 1971. Fungal growth stimulant in anthers which predisposes wheat to attack by Fusarium graminearum. Physiol. Plant Pathol. 1:141-150.

Tag, A. G., Garifullina, G. F., Peplow, A. W., Ake, C., Phillips, T. D., Hohn, T. M., and Beremand, M. N. 2001. A novel regulatory gene, TRI10, controls trichothecene toxin production and gene expression. Appl. Environ. Microbiol. 67:5294-5302.

Tokai, T., Koshino, H., Takahashi-Ando, N., Sato, M., Fujimura, M., and Kimura, M. 2007. Fusarium TRI4 encodes a key multifunctional cytochrome P450 monooxygenase for four consecutive oxygenation steps in trichothecene biosynthesis. Biochem. Biophys. Res. Commun. 353:412417.

U.S. Department of Health and Human Services, Food and Drug Administration 1993. Letter to State Agricultural Directors, State Feed Control Officials, and Food, Feed, and Grain Trade Organizations. Published online.

Voigt, C. A., Schäfer, W., and Salomon, S. 2005. A secreted lipase of Fusarium graminearum is a virulence factor required for infection of cereals. Plant J. 42:364-375.

Voigt, C. A., von Scheidt, B., Gácser, A., Kassner, H., Lieberei, R., Schäfer, W., and Salomon, S. 2007. Enhanced mycotoxin production of a lipase-deficient Fusarium graminearum mutant correlates to toxinrelated gene expression. Eur. J. Plant Pathol. 117:1-12. 